\title{
Syntactic control of concurrency
}

\author{
D.R. Ghica*, A.S. Murawski, C.-H.L. Ong \\ Oxford University Computing Laboratory, Wolfson Building, Parks Road, Oxford OX1 3QD, UK
}

\begin{abstract}
We consider a finitary procedural programming language (finite data-types, no recursion) extended with parallel composition and binary semaphores. Having first shown that may-equivalence of second-order open terms is undecidable we set out to find a framework in which decidability can be regained with minimum loss of expressivity. To that end we define an annotated type system that controls the number of concurrent threads created by terms and give a fully abstract game semantics for the notion of equivalence induced by typable terms and contexts. Finally, we show that the semantics of all typable terms, at any order and in the presence of iteration, has a regular-language representation and thus the restricted observational equivalence is decidable.
\end{abstract}

(C) 2005 Elsevier B.V. All rights reserved.

Keywords: Game semantics; Type systems; Model checking

\section{Introduction}

Game semantics has emerged as a powerful paradigm for giving semantics to a spectrum of programming languages ranging from purely functional languages to those with non-functional features such as control operators and references $[4,19,5,6,21,3]$. Recently, it has been developing in a new, algorithmic direction. Hankin and Malacaria $[23,24]$ have applied it to program analysis. Ghica and McCusker [13] found that the game semantics of a second-order fragment of a procedural language can be captured by regular languages, demonstrating a new, semantics-directed, approach to software model-checking [2]. The approach has subsequently been extended in various directions: to third order [31], call-by-value [10,27], Hoare-style assertions [11] and specifications [12].

In this paper we propose a game-based framework for compositional model checking of concurrent programs. Although a fully abstract game model for a concurrent programming language exists [15], it seems unsuitable as a model of computation for model-checking applications. We can show that observational equivalence, even at second order in the absence of recursion, is not decidable. The sources of non-finitary behaviour are the free identifiers of first or higher-order types, which correspond to procedures using an argument in an unbounded number of concurrent threads of computation.

In the game model, active threads at any moment correspond to pending questions in a play. Hence, we constrain plays by placing bounds on the allowable number of pending questions and enforce these restrictions syntactically using a type system augmented with resource bounds. The key differences between this type system and the standard type system, are the "linearization" of application and parallel composition, i.e. requiring the environments of the two

\footnotetext{
* Corresponding author.

E-mail address: D.R.Ghica@cs.bham.ac.uk (D.R. Ghica).
} 
sub-terms to be disjoint. We also revise the contraction rule to count the number of contracted occurrences of a variable. We call this type system Syntactic Control of Concurrency (SCC); it is a generalization of Serially Reentrant Algol (SRA), a type system introduced by Abramsky [1] to identify higher-order terms of a sequential language denotable by "pointer-free" finitary strategies.

The bounds imposed on the number of pending questions by SCC can be seen as a kind of assume-guarantee reasoning (see e.g. [7]): bounds on the behaviour of the Opponent represent assumptions on the behaviour of the environment, while bounds on the behaviour of the Proponent represent guarantees on the behaviour of the system. Typability can be seen as composition, made possible by the fact that the guarantees and the assumptions match. Unsurprisingly, not all terms of the original language admit a resource-bounding typing.

Resource-sensitive type systems are an area of research with numerous applications; the examples mentioned below are only entry points to a vast literature. The nature of the controlled resource is usually duration [16] or space [17]; applications of such systems are as diverse as execution in embedded systems [18], memory management [33], compilation to hardware [28] or proof-carrying code [29]. Type systems have also been used to control more abstract resources, such as variable usage for improved compilation [35] or interference effects for specification and verification [32].

The motivation behind SCC is to isolate (open) terms with finitary models for the purpose of automated verification. The notion of resource in SCC, which we may call active threads of computation, has a computational meaning, but it is primarily motivated by the game-semantic analysis of the language [15]. The main thrust of the paper is thus semantic; we plan to investigate the type-theoretic issues of SCC separately.

\section{ICA and its game model}

Our object language, Idealized Concurrent Algol (ICA), is Idealized Algol over the finite data-type $\{0, \ldots$, MAX $\}$ $(\mathrm{MAX}>0)$ extended with parallel composition $(\|)$ and binary semaphores. Its types are generated by the grammar given below

$$
\beta::=\operatorname{com}|\exp | \operatorname{var}|\operatorname{sem} \quad \theta::=\beta| \theta \rightarrow \theta
$$

and the typing judgements are displayed in Fig. 1. Semaphores can be manipulated using two (blocking) primitives, $\operatorname{grab}(S)$ and release $(S)$, which grab and, respectively, release the semaphore. We also use variable and semaphore constructors mkvar and mksem (this is necessary for the full abstraction results: Theorems 5 and 19; mkvar was first introduced for this purpose in [5]).

$$
\begin{aligned}
& \Gamma, x: \theta \vdash x: \theta \quad \Gamma \vdash \text { skip : com } \quad \frac{m \in\{0, \cdots, \operatorname{MAX}\}}{\Gamma \vdash m: \exp } \\
& \frac{\Gamma \vdash M: \operatorname{com} \quad \Gamma \vdash N: \operatorname{com}, \exp }{\Gamma \vdash M ; N: \operatorname{com}, \exp } \frac{\Gamma \vdash M: \operatorname{com} \quad \Gamma \vdash N: \text { com }}{\Gamma \vdash M \| N: \operatorname{com}} \\
& \frac{\Gamma \vdash M: \operatorname{var} \quad \Gamma \vdash N: \exp }{\Gamma \vdash M:=N: \operatorname{com}} \quad \frac{\Gamma \vdash M: \operatorname{var}}{\Gamma \vdash ! M: \exp } \\
& \frac{\Gamma, x: \theta \vdash M: \theta^{\prime}}{\Gamma \vdash \lambda x \cdot M: \theta \rightarrow \theta^{\prime}} \frac{\Gamma \vdash M: \theta \rightarrow \theta^{\prime} \quad \Gamma \vdash N: \theta}{\Gamma \vdash M N: \theta^{\prime}} \frac{\Gamma \vdash M: \theta \rightarrow \theta}{\Gamma \vdash \operatorname{fix}(M): \theta} \\
& \frac{\Gamma \vdash M: \exp \quad \Gamma \vdash N_{1}, N_{2}: \beta}{\Gamma \vdash \operatorname{if} M \operatorname{then} N_{1} \text { else } N_{2}: \beta} \frac{\Gamma \vdash M: \exp \quad \Gamma \vdash N: \operatorname{com}}{\Gamma \vdash \text { while } M \operatorname{do} N: \operatorname{com}} \\
& \Gamma \vdash M: \text { sem } \quad \Gamma \vdash M: \text { sem } \\
& \overline{\Gamma \vdash \operatorname{release}(M): \operatorname{com} \quad \Gamma \vdash \operatorname{grab}(M): \operatorname{com}} \\
& \frac{\Gamma, x: \operatorname{var} \vdash M: \operatorname{com}, \exp \quad m \in\left\{0, \cdots, M_{A X}\right\}}{\Gamma \vdash \operatorname{newvar} x:=m \text { in } M: \operatorname{com}, \exp } \\
& \frac{\Gamma, x: \operatorname{sem} \vdash M: \operatorname{com}, \exp \quad m \in\{0, \cdots, \operatorname{MAX}\}}{\Gamma \vdash \operatorname{newsem} x:=m \text { in } M: \operatorname{com}, \exp } \\
& \frac{\Gamma \vdash M: \operatorname{com} \quad \Gamma \vdash N: \operatorname{com}}{\Gamma \vdash \operatorname{mksem} M N: \operatorname{sem}} \frac{\Gamma \vdash M: \exp \rightarrow \operatorname{com} \quad \Gamma \vdash N: \exp }{\Gamma \vdash \operatorname{mkvar} M N: \operatorname{var}}
\end{aligned}
$$

Fig. 1. ICA typing rules. 


$$
\begin{aligned}
& \Sigma \vdash \text { skip } \| \text { skip, } s \longrightarrow \text { skip, } s \\
& \Sigma \vdash \text { skip; } c, s \longrightarrow c, s \\
& \Sigma \vdash \operatorname{newvar} x:=m \text { in } c, s \longrightarrow c, s \\
& \Sigma \vdash \operatorname{newsem} x:=m \text { in } c, s \longrightarrow c, s \\
& \Sigma \vdash \text { if } 0 \text { then } N_{1} \text { else } N_{2}, s \longrightarrow N_{2}, s \\
& \Sigma \vdash \text { if } m \text { then } N_{1} \text { else } N_{2}, s \longrightarrow N_{1}, s, \quad m \neq 0 \\
& \Sigma \vdash ! v, s \otimes(v \mapsto m) \longrightarrow m, s \otimes(v \mapsto m) \\
& \Sigma \vdash v:=m^{\prime}, s \otimes(v \mapsto m) \longrightarrow \operatorname{skip}, s \otimes\left(v \mapsto m^{\prime}\right) \\
& \Sigma \vdash \operatorname{grab}(v), s \otimes(v \mapsto 0) \longrightarrow \operatorname{skip}, s \otimes(v \mapsto 1) \\
& \Sigma \vdash \operatorname{release}(v), s \otimes(v \mapsto m) \longrightarrow \operatorname{skip}, s \otimes(v \mapsto 0), \quad m \neq 0 \\
& \Sigma \vdash(\lambda x . M) N, s \longrightarrow M[N / x], s \\
& \Sigma \vdash \operatorname{fix} M, s \longrightarrow M(\operatorname{fix} M), s \\
& \Sigma \vdash(\operatorname{mkvar} M N):=M^{\prime}, s \longrightarrow M M^{\prime}, s \\
& \Sigma \vdash !(\operatorname{mkvar} M N), s \longrightarrow N, s \\
& \Sigma \vdash \operatorname{grab}(\operatorname{mksem} M N), s \longrightarrow M, s \\
& \Sigma \vdash \operatorname{release}(\operatorname{mksem} M N), s \longrightarrow N, s
\end{aligned}
$$

Fig. 2. Reduction rules for ICA.

The operational semantics is defined using a (small-step) transition relation $\Sigma \vdash M, s \longrightarrow M^{\prime}, s^{\prime}$. $\Sigma$ is a set of names of variables denoting memory cells and those of semaphores denoting locks; $s, s^{\prime}$ are states, i.e. functions $s, s^{\prime}: \Sigma \rightarrow\{0, \ldots, \mathrm{MAX}\}$, and $M, M^{\prime}$ are terms. The basic reduction rules are given in Fig. 2, where $c$ stands for any language constant ( $m$ or skip).

In-context reduction is given by the schemata:

$$
\begin{aligned}
& \frac{\Sigma, v \vdash M[v / x], s \otimes(v \mapsto m) \longrightarrow M^{\prime}, s^{\prime} \otimes\left(v \mapsto m^{\prime}\right) \quad M \neq c}{\Sigma \vdash \operatorname{newvar} x:=m \text { in } M, s \longrightarrow \operatorname{newvar} x:=m^{\prime} \text { in } M^{\prime}[x / v], s^{\prime}}, \\
& \frac{\Sigma, v \vdash M[v / x], s \otimes(v \mapsto m) \longrightarrow M^{\prime}, s^{\prime} \otimes\left(v \mapsto m^{\prime}\right) \quad M \neq c}{\sum \vdash \operatorname{newsem} x:=m \text { in } M, s \longrightarrow \operatorname{newsem} x:=m^{\prime} \text { in } M^{\prime}[x / v], s^{\prime}}, \\
& \frac{\Sigma \vdash M, s \longrightarrow M^{\prime}, s^{\prime}}{\Sigma \vdash \mathcal{E}[M], s \longrightarrow, s^{\prime} \mathcal{E}\left[M^{\prime}\right]},
\end{aligned}
$$

where reduction contexts $\mathcal{E}[-]$ are produced by the grammar:

$$
\begin{aligned}
\mathcal{E}[-]::=[-]|\mathcal{E} ; N|(\mathcal{E} \| N)|(M|| \mathcal{E})| \mathcal{E} N \\
\quad \mid \text { if } \mathcal{E} \text { then } N_{1} \text { else } N_{2}|! \mathcal{E}| \mathcal{E}:=m|M:=\mathcal{E}| \operatorname{grab}(\mathcal{E}) \mid \operatorname{release}(\mathcal{E}) .
\end{aligned}
$$

We consider an angelic notion of termination: we say that a term $M$ terminates in state $s$, written $M, s \Downarrow$, if there exists a terminating evaluation at start state $s: \exists s^{\prime}, M, s \longrightarrow^{*} c, s^{\prime}$, with $c \in\{0, \ldots$, MAX $\}$ or $c=$ skip. If $M$ is closed and $M, \emptyset \Downarrow$ we write $M \Downarrow$. We define the observational approximation relation contextually: $\Gamma \vdash M_{1} \underset{\sim}{\sim} M_{2}$ holds if and only if $\forall \mathcal{C}[-]:$ com, $\mathcal{C}\left[M_{1}\right] \Downarrow$ implies $\mathcal{C}\left[M_{2}\right] \Downarrow$, where $\mathcal{C}\left[M_{i}\right]$ are closed terms of type com. Observational may-equivalence $\left(\Gamma \vdash M_{1} \cong M_{2}\right)$ is then defined as $\Gamma \vdash M_{1} \sqsubset M_{2}$ and $\Gamma \vdash M_{2} \sqsubset M_{1}$.

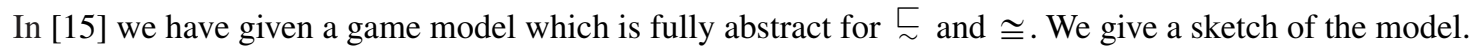

Definition 1. An arena $A$ is a triple $\left\langle M_{A}, \lambda_{A}, \vdash_{A}\right\rangle$ where:

- $M_{A}$ is a set of moves;

- $\lambda_{A}: M_{A} \rightarrow\{O, P\} \times\{Q, A\}$ is a function determining for each $m \in M_{A}$ whether it is an Opponent or a Proponent move, and a question or an answer; we write $\lambda_{A}^{O P}, \lambda_{A}^{Q A}$ for the composite of $\lambda_{A}$ with, respectively, the first and second projections; 
- $\quad{ }_{A}$ is a binary relation on $M_{A}$, called enabling, satisfying: if $m \vdash{ }_{A} n$ for no $m$ then $\lambda_{A}(n)=(O, Q)$, if $m \vdash{ }_{A} n$ then $\lambda_{A}^{O P}(m) \neq \lambda_{A}^{O P}(n)$, and if $m \vdash{ }_{A} n$ then $\lambda_{A}^{Q A}(m)=Q$.

If $m \vdash{ }_{A} n$ we say that $m$ enables $n$. We shall write $I_{A}$ for the set of all moves of $A$ which have no enabler; such moves are called initial. Note that an initial move must be an Opponent question.

The product $(A \times B)$ and arrow $(A \Rightarrow B)$ arenas are defined by

$$
\begin{aligned}
M_{A \times B} & =M_{A}+M_{B}, & M_{A \Rightarrow B} & =M_{A}+M_{B}, \\
\lambda_{A \times B} & =\left[\lambda_{A}, \lambda_{B}\right], & \lambda_{A \Rightarrow B} & =\left[\left\langle\lambda_{A}^{P O}, \lambda_{A}^{Q A}\right\rangle, \lambda_{B}\right], \\
\vdash_{A \times B} & =\vdash_{A}+\vdash_{B}, & \vdash_{A \Rightarrow B} & =\vdash_{A}+\vdash_{B}+\left\{(b, a) \mid b \in I_{B} \text { and } a \in I_{A}\right\},
\end{aligned}
$$

where $\lambda_{A}^{P O}(m)=O$ iff $\lambda_{A}^{O P}(m)=P$. In arenas used to interpret base types all questions are initial and P-moves answer them as detailed in the table below, where $m \in\{0, \ldots$, MAX $\}$.

\begin{tabular}{lccccc}
\hline Arena & O-question & P-answers & Arena & O-question & P-answers \\
\hline$\llbracket \mathbf{c o m} \rrbracket$ & run & $o k$ & $\llbracket \mathbf{e x p} \rrbracket$ & $q$ & $m$ \\
$\llbracket \operatorname{var} \rrbracket$ & read & $m$ & $\llbracket \mathbf{s e m} \rrbracket$ & grab & release \\
& write $(m)$ & $o k$ & & $o k$ \\
\hline
\end{tabular}

A justified sequence in arena $A$ is a finite sequence of moves of $A$ equipped with pointers. The first move is initial and has no pointer, but each subsequent move $n$ must have a unique pointer to an earlier occurrence of a move $m$ such that $m \vdash{ }_{A} n$. We say that $n$ is (explicitly) justified by $m$ or, when $n$ is an answer, that $n$ answers $m$. Note that interleavings of several justified sequences may not be justified sequences; instead we shall call them shuffled sequences. If a question does not have an answer in a justified sequence, we say that it is pending (or open) in that sequence. In what follows we use the letters $q$ and $a$ to refer to question- and answer-moves, respectively, $m$ denotes arbitrary moves. Not all justified sequences are valid. In order to constitute a legal play, a justified sequence must satisfy a well-formedness condition which reflects the "static" style of concurrency of our programming language: any process starting sub-processes must wait for the children to terminate in order to continue. In game terms, if a question is answered then that question and all questions justified by it must have been answered (exactly once). This is spelled out as follows:

Definition 2. The set $P_{A}$ of positions (or plays) over $A$ consists of the justified sequences $s$ over $A$ which satisfy the two conditions below.

FORK: In any prefix $s^{\prime}=\cdots \quad q \longleftarrow m$ of $s$, the question $q$ must be pending before $m$ is played.

WAIT: In any prefix $s^{\prime}=\cdots \quad q \cdots a$ of $s$, all questions justified by $q$ must be answered.

For two shuffled sequences $s_{1}$ and $s_{2}, s_{1} \amalg s_{2}$ denotes the set of all interleavings of $s_{1}$ and $s_{2}$. For two sets of shuffled sequences $S_{1}$ and $S_{2}, S_{1} \amalg S_{2}=\bigcup_{s_{1} \in S_{1}, s_{2} \in S_{2}} s_{1} \amalg s_{2}$. Given a set $X$ of shuffled sequences, we define $X^{0}=X$, $X^{i+1}=X^{i} \amalg X$. Then $X^{\circledast}$, called iterated shuffle of $X$, is defined to be $\bigcup_{i \in \mathbb{N}} X^{i}$.

We say that a subset $\sigma$ of $P_{A}$ is $O$-complete if $s \in \sigma$ and $s o \in P_{A}$, where $o$ is an occurrence of an O-move, entail so $\in \sigma$.

Definition 3. A strategy $\sigma$ on $A$ (written $\sigma: A$ ) is a prefix-closed subset of $P_{A}$, which is $O$-complete.

Strategies $\sigma: A \Rightarrow B$ and $\tau: B \Rightarrow C$ are composed in the standard way, by considering all possible interactions of positions from $\tau$ with shuffled sequences of $\sigma^{\circledast}$ in the shared arena $B$, then hiding the $B$ moves.

The model consists of saturated strategies only: the saturation condition stipulates that all possible (sequential) observations of (parallel) interactions must be present in a strategy: actions of the environment can always be observed earlier if possible, actions of the program can always be observed later. To formalize this, for any arena $A$ a preorder $\preceq$ 
on $P_{A}$ is defined, as the least transitive relation $\preceq$ satisfying $s_{0} \cdot o \cdot s_{1} \cdot s_{2} \preceq s_{0} \cdot s_{1} \cdot o \cdot s_{2}$ and $s_{0} \cdot s_{1} \cdot p \cdot s_{2} \preceq s_{0} \cdot p \cdot s_{1} \cdot s_{2}$ for all $s_{0}, s_{1}, s_{2}$ where $o$ is an O-move and $p$ is a P-move. In the above pairs of positions moves on the left-hand side of $\preceq$ have the same justifiers as on the right-hand side.

Definition 4. A strategy $\sigma$ is saturated iff $s \in \sigma$ and $s^{\prime} \preceq s$ imply $s^{\prime} \in \sigma$.

The two saturation conditions, in various formulations, have a long pedigree in the semantics of concurrency. For example, they have been used by Udding [34] to describe propagation of signals across wires in delay-insensitive circuits and by Josephs et al. [20] to specify the relationship between input and output in asynchronous systems with channels. Laird [22] has been the first to adopt them in game semantics, in his model of Idealized CSP.

Arenas and saturated strategies form a Cartesian closed category $\mathcal{G}_{\text {sat }}$ in which $\mathcal{G}_{\text {sat }}(A, B)$ consists of saturated strategies on $A \Rightarrow B$. The identity strategy is defined by "saturating" the alternating positions $s \in P_{A_{1} \Rightarrow A_{2}}$ such that $\forall t$ 巨even $s, t\left\lceil A_{1}=t\left\lceil A_{2}\right.\right.$, which gives rise to the behaviour of an unbounded buffer (we use $A_{1}$ and $A_{2}$ to distinguish the two copies of $A$ in the arena $A \Rightarrow A$ ).

Other elements of the syntax are interpreted by the least saturated strategies generated by the plays from the table below:

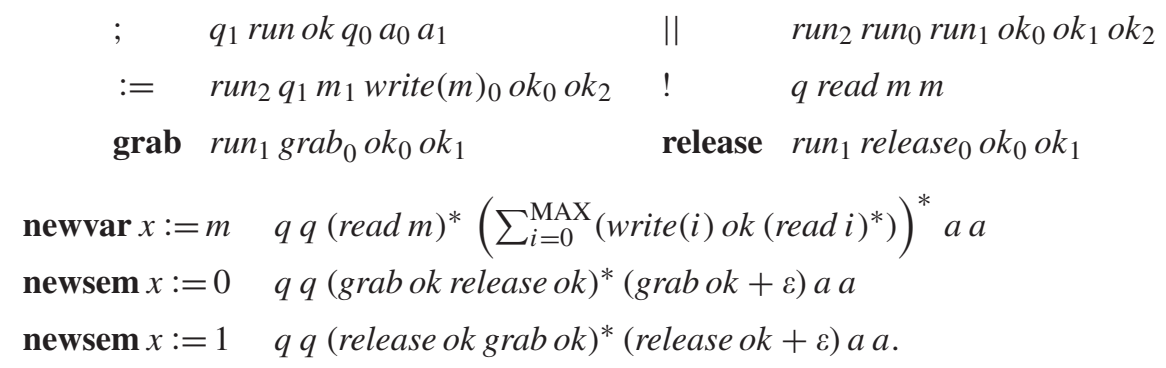

Here we follow a convention (see e.g. [13]) that uses subscripts to distinguish copies of the same move.

As shown in [15], $\mathcal{G}_{\text {sat }}$ is fully abstract for $\cong$ in the sense mentioned below. comp $(\sigma)$ denotes the set of non-empty complete plays of a strategy $\sigma$, i.e. those in which all questions have been answered.

Theorem 5. $\Gamma \vdash M_{1} \underset{\sim}{\sim} M_{2} \Longleftrightarrow \operatorname{comp}\left(\llbracket \Gamma \vdash M_{1} \rrbracket\right) \subseteq \operatorname{comp}\left(\llbracket \Gamma \vdash M_{2} \rrbracket\right)$. Hence, $\Gamma \vdash M_{1} \cong M_{2} \Longleftrightarrow \operatorname{comp}\left(\llbracket \Gamma \vdash M_{1} \rrbracket\right)$ $=\operatorname{comp}\left(\llbracket \Gamma \vdash M_{2} \rrbracket\right)$.

\section{Undecidability of ICA may-equivalence}

A Minsky machine [26] is a state machine with two unbounded counters $c_{1}, c_{2}$. Formally, it can be viewed as a tuple $\left\langle Q, q_{0}, F, \delta\right\rangle$, where $Q$ is the set of states partitioned into disjoint subsets $Q_{1}^{\mathrm{INC}}, Q_{2}^{\mathrm{INC}}, Q_{1}^{\mathrm{DEC}}, Q_{2}^{\mathrm{DEC}}$ with a designated set of final states $F\left(q_{0} \notin F\right)$ and where $\delta$ denotes the two groups of functions: $\delta_{1}: Q_{1}^{\mathrm{INC}} \rightarrow Q, \delta_{2}: Q_{2}^{\mathrm{INC}} \rightarrow Q$ and $\delta_{1}^{0}: Q_{1}^{\mathrm{DEC}} \rightarrow Q, \delta_{2}^{0}: Q_{2}^{\mathrm{DEC}} \rightarrow Q, \delta_{1}^{+}: Q_{1}^{\mathrm{DEC}} \rightarrow Q, \delta_{2}^{+}: Q_{1}^{\mathrm{DEC}} \rightarrow Q$. The machine starts from an initial state $q_{0}$. The initial values of both counters are 0 . When the machine is in state $q \in Q_{i}^{\mathrm{INC}}$, the counter $c_{i}$ is incremented by 1 and the machine moves to $\delta_{i}(q)$. When in state $q \in Q_{i}^{\mathrm{DEC}}$, the next step depends on whether the value of $c_{i}$ is zero. If so, the machine enters $\delta_{i}^{0}(q)$. Otherwise $c_{i}$ is decremented by 1 and the machine moves to $\delta_{i}^{+}(q)$. We say that a Minsky machine machine halts if a final state is entered and the values of both counters are then 0 . It is well-known [26] that the halting problem for Minsky machines is not decidable and we use this to show that neither is observational equivalence.

Theorem 6. ICA may-equivalence is undecidable.

Proof. Let $\Omega_{\text {com }}$ stand for fix $(\lambda x . x)$ : com, i.e. $\Omega_{\text {com }}$ is the divergent command. We show how, given a Minsky machine, one can define a term $g: \mathbf{c o m} \rightarrow \mathbf{c o m} \vdash M:$ com such that $g: \mathbf{c o m} \rightarrow \mathbf{c o m} \vdash M \cong \Omega_{\text {com }}$ if and only if the associated machine does not halt. By Theorem 5 it suffices to show that $\operatorname{comp}(\llbracket g: \mathbf{c o m} \rightarrow \operatorname{com} \vdash M: \operatorname{com} \rrbracket)$ is not empty iff the machine halts. 
The construction of $M$ takes advantage of the fact that the free identifier $g: \mathbf{c o m} \rightarrow \mathbf{c o m}$ represents an indeterminate procedure which can use its argument in a variety of ways (possibly in parallel; the uses may be interleaved or overlapping). This intuition is captured by the use of iterated shuffle in the game model. Using semaphores we are going to restrict the shape of the interleavings (possibly) generated by $g$ in such a way that terminating computations (complete positions) can only arise from a halting run of the Minsky machine.

Let us write $[B]$ for if $B$ then skip else $\Omega_{\text {com }}$. For $i=1,2$ we define the following terms

$$
\begin{aligned}
I_{i} & \equiv \operatorname{grab}(S) ;\left[! S T \in Q_{i}^{\mathrm{INC}}\right] ; S T:=\delta_{i}(! S T) ; \operatorname{release}(S), \\
D_{i}^{+} & \equiv \operatorname{grab}(S) ;\left[! S T \in Q_{i}^{\mathrm{DEC}}\right] ; S T:=\delta_{i}^{+}(! S T) ; \operatorname{release}(S), \\
D_{i}^{0} & \equiv \operatorname{grab}(S) ;\left[! S T \in Q_{i}^{\mathrm{DEC}}\right] ; S T:=\delta_{i}^{0}(! S T) ; \operatorname{release}(S),
\end{aligned}
$$

which will correspond to the three kinds of actions on the counter $c_{i}$. Note that each of the above terms is protected with the same semaphore, so terminating computations can only emerge from interleavings of the six kinds of terms where they are processed in a sequence. Hence, to complete the argument, it suffices to impose additional restrictions guaranteeing that only the sequence simulating actions of the Minsky machine leads to convergence.

We first focus on ensuring that zero tests (modelled by $D_{i}^{+}$or $D_{i}^{0}$ ) are handled correctly. By a simple history of a counter we mean a (possibly empty) series of increments and decrements starting at value 0 resulting in value 0 , possibly followed by a zero test. Observe that the full history of a counter in a halting Minsky machine is a sequence of simple histories. Then the term

$$
C_{i} \equiv \operatorname{grab}\left(S_{i}\right) ; g\left(I_{i} ; D_{i}^{+}\right) ;\left(D_{i}^{0} \text { or skip }\right) ; \operatorname{release}\left(S_{i}\right)
$$

corresponds to all simple histories of $c_{i}$ : because copies of $I_{i}$ and $D_{i}^{+}$will never overlap in terminating computations, $g\left(I_{i} ; D_{i}^{+}\right)$corresponds to all potential sequences of increments and decrements of $c_{i}$ leading from value 0 to 0 . Note also the use of semaphores $S_{i}$ which will guarantee that different copies of $C_{1}$ ( $C_{2}$, respectively), i.e. simple histories of each counter, can be interleaved only sequentially, while the sequence of $C_{1}$ 's and the sequence of $C_{2}$ 's can interact freely. This ensures that $g\left(C_{1}\right.$ or $\left.C_{2}\right)$, where

$$
C_{1} \text { or } C_{2} \equiv \text { newvar } x \text { in }(x:=0 \| x:=1) \text {; if } ! x \text { then } C_{1} \text { else } C_{2},
$$

will represent all sequences of $I_{1}, I_{2}, D_{1}^{+}, D_{1}^{0}, D_{2}^{+}, D_{2}^{0}$ in which before each $D_{i}^{+}$the number of $I_{i}$ 's always exceeds that of $D_{i}^{+}$'s and before each $D_{i}^{0}$ we have an equal number of $I_{i}$ 's and $D_{i}^{+}$'s.

Finally, in order to capture a halting run of the given Minsky machine, we have to make sure that the sequences above are consistent with state changes of the machine. This is however already guaranteed by assertions of the form [!ST $\in \cdots]$ in $I_{i}, D_{i}^{+}, D_{i}^{0}$ whose presence we have ignored in our argument so far. $M$ can thus be taken to be

$$
\begin{aligned}
& g: \text { com } \rightarrow \text { com } \vdash \quad \text { newvar } S T:=q_{0} \text { in } \\
& \text { newsem } S, S_{1}, S_{2}:=0,0,0 \text { in } g\left(C_{1} \text { or } C_{2}\right) ;[! S T \in F] .
\end{aligned}
$$

\section{SCC: a resource-bounding type system}

The simulation above is possible because free identifiers com $\rightarrow$ com correspond to functions that investigate the argument an arbitrary number of times (possibly in parallel). Therefore, the key to regaining decidability is to restrict the number of times an argument is used concurrently. However, we need not restrict the number of sequential uses, to allow for iteration and all sorts of interesting procedural programs.

The type system is for the recursion-free fragment with while. Divergence, $\Omega_{\mathbf{c o m}}$, can then be defined by while 1 do skip. Types are generated by the following grammar:

$$
\beta::=\operatorname{com}|\exp | \operatorname{var}|\mathbf{s e m} \quad \theta::=\beta| \gamma \rightarrow \theta \quad \gamma::=\theta^{n} .
$$

The numbers that label the left-hand side of a function type will be called resource bounds. An occurrence $m$ of a resource bound in a type $\theta$ is an assume (respectively, guarantee) if it occurs in the left-hand scope of an even (respectively, 
odd) number of $\rightarrow$ 's in $\theta$. Formally, $m$ is an assume (a guarantee) in $\theta$ iff $\theta=\mathcal{A}[m](\theta=\mathcal{G}[m])$ :

$$
\begin{aligned}
& \left.\mathcal{G}[]::=\theta_{1}^{[}\right] \rightarrow \theta_{2}\left|\theta^{n} \rightarrow \mathcal{G}[]\right| \mathcal{A}[]^{n} \rightarrow \theta, \\
& \mathcal{A}[]::=\theta^{n} \rightarrow \mathcal{A}[] \mid \mathcal{G}[]^{n} \rightarrow \theta .
\end{aligned}
$$

For instance, 3 in $\left(\mathbf{c o m}^{3} \rightarrow \mathbf{c o m}\right)^{4} \rightarrow \mathbf{c o m}$ is an assume and 4 is a guarantee. Assumes and guarantees will turn out to correspond to the Opponent/Player polarity in game semantics.

Assumes characterize the behaviour of the program context and guarantees characterize that of the program. The assumes of a typing judgement $\theta_{1}^{n_{1}}, \ldots, \theta_{k}^{n_{k}} \vdash M: \theta$ are the assumes in $\theta$ along with the guarantees in $\theta_{1}, \ldots, \theta_{k}$. The guarantees of a typing judgement are the guarantees of $\theta$, the assumes in $\theta_{1}, \ldots, \theta_{k}$ and $n_{1}, \ldots, n_{k}$.

We use types of this form to approximate the maximum number of concurrent sub-threads of computation at any moment. This estimate is subject to assumes on the environment. Intuitively, if a program has a type $\theta$, then provided the environment behaves according to the assumes, the program's behaviour satisfies the guarantees. In this spirit we introduce a sub-typing relation which can be taken to correspond to weakening the constraints imposed by SCC.

$$
\beta \leqslant \beta \quad \frac{n_{1} \leqslant n_{2}}{\theta^{n_{2}} \leqslant \theta^{n_{1}}} \quad \frac{\gamma_{2} \leqslant \gamma_{1} \quad \theta_{1} \leqslant \theta_{2}}{\gamma_{1} \rightarrow \theta_{1} \leqslant \gamma_{2} \rightarrow \theta_{2}} .
$$

Intuitively, a subtype gives a less precise approximation: higher on the behaviour of the program and lower for the environment. In the latter case, the bound is considered inferior because it applies to a weaker behaviour of the environment.

The SCC typing rules are given in Fig. 3. Typing judgements are of the form $\Gamma \vdash_{r} M: \theta$, where $\Gamma=x_{1}: \theta_{1}^{n_{1}}, \ldots, x_{k}: \theta_{k}^{n_{k}}$; we write $n \Gamma=x_{1}: \theta_{1}^{n \cdot n_{1}}, \ldots, x_{k}: \theta_{k}^{n \cdot n_{k}}$. Note that the typing rules make a distinction between parallel and sequential composition. Parallel composition and application have multiplicative rules, in which the contexts are required to be disjoint, as opposed to the rules for sequential operators ( $\square$ can stand for ; , :=, !, grab, release) including branching and iteration. In order to be able to use identifiers (e.g. semaphores) in concurrent threads, a contraction rule is necessary; we modify it so that the guaranteed bounds on the contracted variables are accumulated into the new variable.

Remark 7. The rule for application is also multiplicative. The reason is that call-by-name application is a peculiar form of concurrency in which the computation carried out by the function is interleaved with that of its argument, albeit in a highly constrained fashioned. For instance, if $F$ is a first-order function, any computation arising in an application $F(M)$ also arises in the parallel composition $\cdots F(\cdots) \cdots\|\cdots M \cdots\| \cdots \| \cdots M$, where the ellipses stand for code manipulating semaphores so that the right interleaving of effects is enforced [15]. A multiplicative application rule is also used in SRA [1].

Example 8. For any $n \in \mathbb{N}$ we have

(1) $\vdash_{r} \lambda f x . f(f(x)):\left(\mathbf{c o m}^{n} \rightarrow \mathbf{c o m}\right)^{n+1} \rightarrow\left(\operatorname{com}^{n^{2}} \rightarrow \mathbf{c o m}\right)$

(2) $\vdash_{r} \lambda f x . f(x) ; f(x):\left(\mathbf{c o m}^{n} \rightarrow \mathbf{c o m}\right)^{1} \rightarrow\left(\operatorname{com}^{n} \rightarrow \mathbf{c o m}\right)$

(3) $\vdash_{r} \lambda f x . f(x) \| f(x):\left(\mathbf{c o m}^{n} \rightarrow \mathbf{c o m}\right)^{2} \rightarrow\left(\operatorname{com}^{2 n} \rightarrow \mathbf{c o m}\right)$

(4) $\vdash_{r} \lambda f . f(f$ skip $):\left(\mathbf{c o m}^{n} \rightarrow \mathbf{c o m}\right)^{n+1} \rightarrow \mathbf{c o m}$

(5) $\vdash_{r} \lambda g . g(\lambda x . g(\lambda y . x)):\left(\left(\operatorname{com}^{n} \rightarrow \mathbf{c o m}\right)^{n} \rightarrow \mathbf{c o m}\right)^{n+1} \rightarrow \mathbf{c o m}$.

SCC enjoys the standard syntactic properties of a typed lambda calculus (basis, generation, sub-term, substitution and subject reduction lemmas) [8]. We also have the easily derivable dual of the subsumption law:

$$
\frac{\Gamma, x: \theta^{\prime} \vdash_{r} M: \theta^{\prime \prime} \quad \theta \leqslant \theta^{\prime}}{\Gamma, x: \theta \vdash_{r} M: \theta^{\prime \prime}} .
$$

Not all ICA terms are typable in SCC. However, if one attempts to prove typability by induction on the structure of ICA derivations, it turns out that only the application rule does not preserve it. This is because the corresponding SCC rule requires that the bounds of the argument match those of the function term. For example, the application of the term 5 to term 4 above is untypable.

Given the bounds for environment, SCC can be used to certify bounds for the program. 
Axioms:

$$
x: \theta^{1} \vdash_{r} x: \theta \quad \vdash_{r} \text { skip : com } \quad \frac{m \in\{0, \cdots, \operatorname{MAX}\}}{\vdash_{r} m: \exp }
$$

Additive rules:

$$
\begin{aligned}
& \frac{\Gamma \vdash_{r} M: \theta}{\Gamma, x: \gamma \vdash_{r} M: \theta} \quad \frac{\Gamma \vdash_{r} M: \theta \quad \theta \leq \theta^{\prime}}{\Gamma \vdash_{r} M: \theta^{\prime}} \\
& \frac{\left\{\Gamma \vdash_{r} M: \theta_{1}\right\} \quad \Gamma \vdash_{r} N: \theta_{2}}{\Gamma \vdash_{r}\{M\} \square N: \theta_{3}} \quad \frac{\Gamma, x: \gamma \vdash_{r} M: \theta}{\Gamma \vdash_{r} \lambda x \cdot M: \gamma \rightarrow \theta} \\
& \frac{\Gamma \vdash_{r} M: \exp \quad \Gamma \vdash_{r} N_{1}, N_{2}: \beta}{\Gamma \vdash_{r} \text { if } M \text { then } N_{1} \text { else } N_{2}: \beta} \quad \frac{\Gamma \vdash_{r} M: \exp \quad \Gamma \vdash_{r} N: \text { com }}{\Gamma \vdash_{r} \text { while } M \text { do } N: \text { com }} \\
& \Gamma, x: \operatorname{var}^{n} \vdash_{r} M: \mathbf{c o m}, \exp \\
& \Gamma \vdash_{r} \text { newvar } x:=m \text { in } M: \operatorname{com}, \exp \\
& \Gamma, x: \mathbf{s e m}^{n} \vdash_{r} M: \mathbf{c o m}, \mathbf{e x p} \\
& \bar{\Gamma} \vdash_{r} \text { newsem } x:=m \text { in } M: \operatorname{com}, \exp \\
& \frac{\Gamma \vdash_{r} M: \text { com } \quad \Gamma \vdash_{r} N: \text { com }}{\Gamma \vdash_{r} \text { mksem } M N: \text { sem }} \\
& \frac{\Gamma \vdash_{r} M: \exp ^{n} \rightarrow \operatorname{com} \quad \Gamma \vdash_{r} N: \exp }{\Gamma \vdash_{r} \operatorname{mkvar} M N: \operatorname{var}}
\end{aligned}
$$

Multiplicative rules:

$$
\begin{gathered}
\frac{\Gamma, x: \theta^{n_{1}}, y: \theta^{n_{2}} \vdash_{r} M: \theta^{\prime}}{\Gamma, x: \theta^{n_{1}+n_{2}} \vdash_{r} M[x / y]: \theta^{\prime}} \quad \frac{\Gamma \vdash_{r} M: \operatorname{com} \Delta \vdash_{r} N: \text { com }}{\Gamma, \Delta \vdash_{r} M \| N: \text { com }} \\
\frac{\Gamma \vdash_{r} M: \theta^{n} \rightarrow \theta^{\prime} \Delta \vdash_{r} N: \theta}{\Gamma, n \Delta \vdash_{r} M N: \theta^{\prime}}
\end{gathered}
$$

Fig. 3. SCC typing rules.

Definition 9. An ICA term $\Gamma \vdash M: \theta$ is $r$-typable if for any assignment of assumes there exists an assignment of guarantees such that when we adorn $\Gamma, \theta$ with these bounds we get $\Gamma^{\prime}, \theta^{\prime}$ such that $\Gamma^{\prime} \vdash_{r} M: \theta^{\prime}$. We shall write $\eta^{\mathrm{a}}, \eta^{\mathrm{g}}$, respectively, for the two assignments.

Since not all terms are typable, not all terms are $r$-typable. However, there is a wide class of $r$-typable (and so typable) terms. The key to regaining typability lies in restricting the shape of possible applications. The lemma below exhibits two instances where typability is preserved (a combination of the two is also possible).

Lemma 10. (1) Any $\beta$-normal ICA term is r-typable.

(2) Any ICA term in which function arguments are of first order or base type is r-typable.

Proof. We reason by induction on the ICA typing rules. All cases except the application rule are routine appeals to the induction hypothesis. For rules with more than one premise it is necessary to apply the dual law given above to find a common typing (by using the higher of the guarantees provided by the several appeals to the induction hypothesis). Additionally, for $\|$, contraction has to be used. Finally, we consider the restricted forms of application.

(1) If a term is $\beta$-normal then all applications have the form $\Gamma \vdash f M_{1} \cdots M_{k}$. For simplicity, we will assume that $k=1$ (the argument for $k>1$ follows the same pattern).

Suppose $\Gamma \vdash f M_{1}: \theta_{2}$ and $\eta^{\mathrm{a}}$ is an assignment of assumes to the typing judgement. Let $\eta_{r}^{\mathrm{a}}=\eta^{\mathrm{a}} \uparrow \theta_{2}$. Note that $f: \theta_{1} \rightarrow \theta_{2}$, for some $\theta_{1}$, must be present in $\Gamma$, so $\eta^{\text {a }}$ also defines bounds for the associated occurrence of $\theta_{1} \rightarrow \theta_{2}$. Let $\eta_{f}^{\mathrm{a}}=\eta^{\mathrm{a}} \uparrow\left(\theta_{1} \rightarrow \theta_{2}\right)$. 
Consider $\Gamma \vdash M_{1}: \theta_{1}$ and the assignment of assumes in which the bounds for $\Gamma$ are the same as in $\eta^{\text {a }}$ and those for $\theta_{1}$ are determined by $\eta_{f}^{\mathrm{a}}$. By IH we get $\Gamma^{\prime} \vdash_{r} M_{1}: \theta_{1}^{\prime}$. Define the resource type $\theta_{2}^{\prime}$ by decorating $\theta_{2}$ with assumes given by $\eta_{r}^{\mathrm{a}}$ and guarantees which are assumes in $\eta_{f}^{\mathrm{a}}$. Then we have $f:\left(\theta_{1}^{\prime} \rightarrow \theta_{2}^{\prime}\right)^{1} \vdash_{r} f:\left(\theta_{1}^{\prime} \rightarrow \theta_{2}^{\prime}\right)$ and, consequently, $f:\left(\theta_{1}^{\prime} \rightarrow \theta_{2}^{\prime}\right), \Gamma^{\prime} \vdash_{r} f M_{1}: \theta_{2}^{\prime}$. Recall that $\Gamma^{\prime}$ will contain an occurrence of $f$ where the associated assumes are the same as those for $f$. Using the dual subsumption law we can make the guarantees match and finish by contracting $f$.

(2) Suppose $\Gamma \vdash M N: \theta$ and $\Gamma \vdash N: \beta \rightarrow \beta$. Let $\eta^{\text {a }}$ be an assignment of assumes to the first judgement. By $\mathrm{IH}$, using assumes from $\eta^{\text {a }}$ for $\Gamma$, we have $\Gamma^{\prime} \vdash_{r} N: \beta^{n^{\prime}} \rightarrow \beta$. Now consider $\Gamma \vdash M:(\beta \rightarrow \beta) \rightarrow \theta$. By IH, using assumes from $\eta^{\text {a }}$ for $\Gamma$ and $n^{\prime}$ for the leftmost occurrence of $\beta$, we get $\Gamma^{\prime \prime} \vdash_{r} M:\left(\beta^{n^{\prime}} \rightarrow \beta\right)^{n} \rightarrow \theta^{\prime}$. Hence, $\Gamma^{\prime \prime}, n \Gamma^{\prime} \vdash_{r} M N: \theta^{\prime}$. Because $\Gamma^{\prime \prime}$ and $\Gamma^{\prime}$ share the same assumes, we can unify the guarantees inside $\Gamma^{\prime}$ and $\Gamma^{\prime \prime}$ using the dual subsumption law and follow with contraction to get $\Gamma^{\prime \prime \prime} \vdash_{r} f M: \theta^{\prime}$, where the assumes in $\Gamma^{\prime \prime \prime}$ coincide with $\eta^{\mathrm{a}}$.

Using SCC we can define a new observational approximation relation $\check{\sim}_{r}$ using typable terms and contexts along with their bounds. Suppose $\Gamma \vdash_{r} M_{1}, M_{2}: \theta$. In what follows we write $\vdash_{r} \mathcal{C}\left[M_{i}\right]$ to mean that $\mathcal{C}\left[M_{i}\right]$ is typable and its derivation is constructed using (possibly several copies of) the given derivation of the typing judgement $\Gamma \vdash_{r} M_{i}$, up to appropriate renaming of variables. We define $\Gamma \vdash_{r} M_{1} \check{r}_{r} M_{2}$ to hold iff for all contexts $\mathcal{C}[-]$ such that $\vdash_{r} \mathcal{C}\left[M_{i}\right]:$ com we have: $\mathcal{C}\left[M_{1}\right] \Downarrow$ implies $\mathcal{C}\left[M_{2}\right] \Downarrow$. Similarly, we write $\Gamma \vdash_{r} M_{1} \cong{ }_{r} M_{2}$ iff $\Gamma \vdash_{r} M_{1} \sim{ }_{r} M_{2}$ and $\Gamma \vdash_{r} M_{2} \underset{\sim}{\sim} M_{1}$. In particular, the definition applies to the terms for which the above lemma holds. Note that no bound needs to be placed on the way $M_{i}$ is used in $\mathcal{C}\left[M_{i}\right]$, the bounds concern only the way its free identifiers are trapped in context. In the definition of $\check{\sim}_{r}$ we require $\Gamma \vdash_{r} M_{i}: \theta$ to have the same annotations. If two terms are typable with the same assumed bounds, it is always possible to type them with the same guaranteed bounds by sub-typing.

Example 11 (Brookes [9]). Consider the terms

$$
\begin{aligned}
& M_{1} \equiv \text { newvar } x:=0 \text { in }\left(p(x:=! x+1 ; x:=! x+1) ; \text { if } \text { even }(! x) \text { then } \Omega_{\text {com }}\right) \\
& M_{2} \equiv \text { newvar } x:=0 \text { in }\left(p(x:=! x+2) ; \text { if } \text { even }(! x) \text { then } \Omega_{\text {com }}\right)
\end{aligned}
$$

with $p: \mathbf{c o m} \rightarrow \mathbf{c o m}$. Brookes has shown that in sequential Algol they are observationally equivalent, whereas in concurrent Algol they are not. In SCC we have $p:\left(\mathbf{c o m}^{1} \rightarrow \mathbf{c o m}\right)^{1} \vdash_{r} M_{1} \cong_{r} M_{2}$; but for any (assumed) bound $n>1, p:\left(\mathbf{c o m}^{n} \rightarrow \mathbf{c o m}\right)^{1} \vdash_{r} M_{1} \varlimsup_{r} M_{2}$. The reason is that the assumed bound of 1 only allows identifier $p$ to be bound to a procedure which uses its argument sequentially. For example, context $\mathcal{C}[-]=(\lambda p .[-])(\lambda c . c \| c)$ cannot trap $p: \operatorname{com}^{1} \rightarrow \mathbf{c o m}$. On the other hand, context $\mathcal{C}[-]=(\lambda p .[-])(\lambda c . c ; c)$ can trap $p: \mathbf{c o m}^{n} \rightarrow \mathbf{c o m}$ for any $n$.

A formal proof of this example is immediate once the connection with game semantics is established in the following section.

\section{The game model revisited}

We use the game model to interpret the annotations from the type system and to show how the model can be used to reason about $\check{\sim}_{r}, \cong_{r}$. In order to analyse the positions induced by terms in more detail we define a more refined games framework where plays can form a subset of $P_{A}$ as opposed to the full $P_{A}$. In particular, we are going to dissect the possibilities for the function space game $A \Rightarrow B$. To do that we introduce an auxiliary notion of games in which shuffled sequences are allowed (cf. [25]).

Definition 12. A bounded game $\underline{A}$ is a pair $\left\langle A, R_{A}\right\rangle$ where $A$ is an arena and $R_{A}$ is a prefix-closed subset of $P_{A}^{\circledast}$.

We also refer to the elements of $R_{A}$ as plays and write $\operatorname{comp}\left(R_{A}\right)$ for the set of complete plays in $R_{A}$ (those in which all questions are answered). The games of $\mathcal{G}_{\text {sat }}$ can be viewed as bounded games where $R_{A}=P_{A}$. 
Using bounded games we can define a more refined type hierarchy:

$$
\begin{aligned}
\underline{A} \times \underline{B} & =\left(A \times B, R_{A}+R_{B}\right) \\
\underline{A} \otimes \underline{B} & =\left(A \times B, R_{A} \amalg R_{B}\right) \\
! \underline{A} & =\left(A, R_{A}^{\circledast}\right) \\
\underline{A} \multimap \underline{B} & =\left(A \Rightarrow B,\left\{s \in P_{A \Rightarrow B}^{\circledast} \mid s \uparrow A \in R_{A}, s\left\lceil B \in R_{B}\right\}\right) .\right.
\end{aligned}
$$

We can then construct an arrow type as

$$
\underline{A} \Rightarrow \underline{B}=! \underline{A} \multimap \underline{B} .
$$

We also have

$$
! \underline{A} \otimes ! \underline{B}=!(\underline{A} \times \underline{B}) .
$$

Note that where $R_{A}=P_{A}, R_{B}=P_{B}$ the $\times$ and $\Rightarrow$ constructions coincide with the previous ones.

Let us now define

$$
! \underline{A}=\left(A,\left(\operatorname{comp}\left(R_{A}\right)\right)^{*} \cdot R_{A}\right),
$$

i.e. ! is an impoverished, sequential, version of ! where a new "thread" of $R_{A}$ can be started only when the previous one is completed. Obviously, $R_{!_{\underline{A}}} \subseteq R_{! \underline{A}}$.

An important case of $\varrho \underline{A}$, which we use in the following, is when $\underline{A}$ is well-opened, i.e. each play in $R_{A}$ can contain only one occurrence of an initial move, namely, the first move of the play (all games interpreting ICA types are of that kind). Then $! \underline{A}$ contains plays which might have many occurrences of initial moves, but only one occurrence of an initial question can be open (pending) at any time. Similarly, $\bigotimes_{1 \leqslant i \leqslant n} \underline{! A}$ contains plays with at most $n$ pending questions; we shall write $\underline{A}^{n}$ for it. We use this construction to specify restricted function spaces: instead of $\underline{A} \Rightarrow \underline{B}=! \underline{A} \multimap \underline{B}$ we consider $\underline{A}^{n} \multimap \underline{B}$. These restrictions turn out to give the correct interpretation of the bounds inferred by the type system for SCC.

Regardless of whether we deal with standard ICA type or typing judgements (annotated with bounds or not) $\llbracket \cdots \rrbracket$ stands for the usual interpretation in $\mathcal{G}_{\text {sat }}$ (i.e. the information about bounds is completely ignored by $\llbracket \cdots \rrbracket$ ). We introduce the notation $\llbracket \cdots \rrbracket_{\eta}$ for bound-sensitive semantic interpretation.

Let $\Gamma \vdash_{r} M: \theta$, where $\Gamma=\theta_{1}^{n_{1}}, \ldots, \theta_{k}^{n_{k}}$. In $\mathcal{G}_{\text {sat }}$ it is standardly interpreted by a strategy for the game $\llbracket \Gamma \vdash \theta \rrbracket=$ $\llbracket \theta_{1} \rrbracket \times \cdots \times \llbracket \theta_{k} \rrbracket \Rightarrow \llbracket \theta \rrbracket$ or, equivalently, $! \llbracket \theta_{1} \rrbracket \otimes \cdots \otimes ! \llbracket \theta_{k} \rrbracket \multimap \llbracket \theta \rrbracket$. Suppose $\eta$ represents a vector of resource bounds consistent with $\Gamma \vdash_{r} M: \theta$. It is not necessary that $\eta$ includes all the bounds used in the resource-sensitive type judgement. Then the corresponding bounded game, denoted by $\llbracket \Gamma \vdash \theta \rrbracket_{\eta}$, is defined inductively in the same way as $\llbracket \Gamma \vdash \theta \rrbracket$ except that whenever a bound $n$ is specified by $\eta$ (for an occurrence of $\rightarrow$ or $\theta_{i}$ ), we use $A^{n} \multimap B$ and $A^{n}$ instead of, respectively, $A \Rightarrow B=! A \multimap B$ and $! A$.

Example 13. Suppose we have $x_{1}:\left(\mathbf{c o m}^{9} \rightarrow \mathbf{s e m}\right)^{5}, x_{2}:\left(\exp ^{3} \rightarrow \mathbf{c o m}\right)^{7} \vdash M: \exp ^{7} \rightarrow$ var. The complete vector of resource bounds is $(9,5,3,7,7)$. Let $\eta$ stand for the distinguished bounds $(-, 5,3,-, 7)$. Then

$$
\begin{aligned}
& \llbracket \operatorname{com} \rightarrow \text { sem }, \exp \rightarrow \operatorname{com} \vdash \exp \rightarrow \operatorname{var} \rrbracket_{\eta} \\
& \quad=(! \llbracket \operatorname{com} \rrbracket \multimap \llbracket \operatorname{sem} \rrbracket)^{5} \otimes !\left(\llbracket \exp \rrbracket^{3} \multimap \llbracket \operatorname{com} \rrbracket\right) \multimap\left(\llbracket \exp \rrbracket^{7} \multimap \llbracket \operatorname{var} \rrbracket\right) .
\end{aligned}
$$

This notation is flexible enough to handle assumes, guarantees or combined assume-guarantee resource bounds in a uniform way.

Now we are ready to interpret the bounds given by the type system using the game model. We denote the interpretation by $\llbracket \Gamma \vdash M: \theta \rrbracket_{\eta^{\mathrm{a}}}$. It is simply $\llbracket \Gamma \vdash M: \theta \rrbracket$ in which O-moves are restricted to those allowed by the $A^{n} \multimap B$ games consistent with the bounds in $\eta^{\mathrm{a}}$ :

$$
\llbracket \Gamma \vdash M: \theta \rrbracket_{\eta^{\mathrm{a}}}=\llbracket \Gamma \vdash M: \theta \rrbracket \cap R_{\llbracket \Gamma \vdash \theta \rrbracket_{\eta^{\mathrm{a}}}} .
$$


More precisely, for each occurrence $m$ of an initial move from such $B$ Opponent will not be allowed to play an initial move from $A$ justified by $m$ if the current position already contains $n$ pending questions justified by $m$. The guaranteed bounds given by SCC are then sound in that they are correct approximations of the shape of positions explored by $\mathrm{P}$

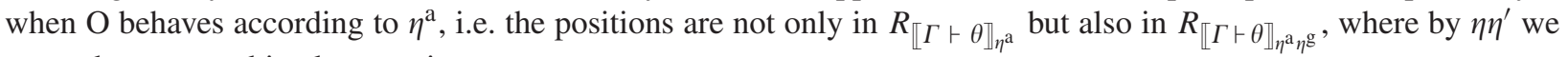
mean the two combined constraint vectors.

Theorem 14. $\llbracket \Gamma \vdash M: \theta \rrbracket_{\eta^{\mathrm{a}}} \subseteq R_{\llbracket \Gamma \vdash \theta \rrbracket_{\eta^{\mathrm{a}} \eta^{\mathrm{g}}}}$.

Proof. The theorem can be proved by induction on the derivation of $\Gamma \vdash_{r} M: \theta$. As before, application is, technically, the most difficult. In all other cases it is easy to see that the definition of $\llbracket \Gamma \vdash M: \theta \rrbracket_{\eta^{\mathrm{a}}}$ is compositional: $\llbracket \Gamma \vdash M: \theta \rrbracket_{\eta^{\mathrm{a}}}$ can be defined directly by induction on the structure of $\vdash_{r}$ derivations. Consequently, a simple appeal to the induction hypothesis does the job.

For application, let $\eta_{1}^{\mathrm{a}}, \eta_{2}^{\mathrm{a}}, \eta_{3}^{\mathrm{a}}$ represent the assumed bounds of the respective three judgements:

$$
\frac{\Gamma \vdash_{r} M: \theta^{n} \rightarrow \theta^{\prime} \quad \Delta \vdash_{r} N: \theta}{\Gamma, n \Delta \vdash_{r} M N: \theta^{\prime}} .
$$

Let us make the following definitions:

$$
\begin{aligned}
\sigma_{1} & =\llbracket \Gamma \vdash M: \theta \rightarrow \theta^{\prime} \rrbracket_{\eta_{1}^{\mathrm{a}}}, & \sigma_{1}^{\prime}=\llbracket \Gamma \vdash M: \theta \rightarrow \theta^{\prime} \rrbracket_{\eta_{3}^{\mathrm{a}} \mid \Gamma, \theta^{\prime}}, \\
\sigma_{2} & =\llbracket \Delta \vdash N: \theta \rrbracket_{\eta_{2}^{\mathrm{a}}}, & \sigma_{2}^{\prime}=\llbracket \Delta \vdash N: \theta \rrbracket_{\eta_{3}^{\mathrm{a}} \backslash \Delta},
\end{aligned}
$$

where we write $\eta\left\lceil\Theta\right.$ for $\eta$ restricted to a list of types $\Theta$. The only difference between $\sigma_{i}$ and $\sigma_{i}^{\prime}(i=1,2)$ is that in $\sigma_{i}^{\prime}$ there are no bounds on O-moves in $\theta$. Otherwise, they are subject to the same restrictions, because $\eta_{1}^{\mathrm{a}} \uparrow \Gamma, \theta^{\prime}=\eta_{3}^{\mathrm{a}} \uparrow \Gamma, \theta^{\prime}$ and $\eta_{2}^{\mathrm{a}} \uparrow \Delta=\eta_{3}^{\mathrm{a}}\lceil\Delta$.

Consider $\llbracket \Gamma, n \Delta \vdash M N: \theta^{\prime} \rrbracket_{\eta_{3}^{\mathrm{a}}}$, which is defined by interactions of $\sigma_{1}^{\prime}$ with $\sigma_{2}^{\prime}$. Note that up to the first move in $\theta$, $\sigma_{1}^{\prime}$ behaves in the same way as $\sigma_{1}$. Then, as $\sigma_{1}^{\prime}$ and $\sigma_{2}^{\prime}$ interact, the induction hypotheses imply that the guarantees provided by each of the strategies match the assumed bounds of the other $\left(\eta_{1}^{\mathrm{g}} \uparrow \theta=\eta_{2}^{\mathrm{a}}\left\lceil\theta, \eta_{2}^{\mathrm{g}} \uparrow \theta=\eta_{1}^{\mathrm{a}}\lceil\theta)\right.\right.$. Thus, the interaction of $\sigma_{1}^{\prime}$ and $\sigma_{2}^{\prime}$ is actually constrained to positions of $\sigma_{1}$ and $\sigma_{2}$. Consequently, $\llbracket \Gamma, n \Delta \vdash M N: \theta^{\prime} \rrbracket \eta_{3}^{\text {a }}$ can also be defined compositionally by interactions of $\sigma_{1}$ and $\sigma_{2}$ and the rest easily follow.

The sets of complete plays induced by the restricted denotations comp $\left(\llbracket \Gamma \vdash_{r} M: \theta \rrbracket_{\eta^{a}}\right)$ turn out to provide a fully abstract model of $\sqsubset_{r}$.

Lemma 15. Suppose $\Gamma \vdash_{r} M_{1}, M_{2}: \theta$ and let $\eta^{\mathrm{a}}$ be the final assignment of assumed bounds. Then comp $\left(\llbracket \Gamma \vdash_{r} M_{1}\right.$ : $\left.\theta \rrbracket_{\eta^{\mathrm{a}}}\right) \subseteq \operatorname{comp}\left(\llbracket \Gamma \vdash_{r} M_{2}: \theta \rrbracket_{\eta^{\mathrm{a}}}\right)$ implies $\Gamma \vdash M_{1} \underset{\sim}{\sim} M_{2}: \theta$.

Proof. Suppose $\vdash_{r} C\left[M_{i}\right]$ : $\operatorname{com}(i=1,2)$ and $C\left[M_{1}\right] \Downarrow$. Then, by the soundness of $\mathcal{G}_{\text {sat }}[15], \operatorname{comp}\left(\llbracket C\left[M_{1}\right] \rrbracket\right) \neq \emptyset$. As noted in the proof of Theorem 14, $\llbracket C\left[M_{1}\right] \rrbracket$ can be defined inductively through $\llbracket \Gamma \vdash M_{1} \rrbracket \eta^{\text {a }}$ so, because $\operatorname{comp}\left(\llbracket \Gamma \vdash_{r} M_{1} \rrbracket_{\eta^{\mathrm{a}}}\right) \subseteq \operatorname{comp}\left(\llbracket \Gamma \vdash_{r} M_{2} \rrbracket_{\eta^{\mathrm{a}}}\right)$, we also have comp $\left(\llbracket C\left[M_{2}\right] \rrbracket\right) \neq \emptyset$. Thus, again by the adequacy of $\mathcal{G}_{\text {sat }}, C\left[M_{2}\right] \Downarrow$ and indeed $M_{1} \underset{r}{\sim} M_{2}$.

To prove the converse we need to strengthen the definability result from [14] to ensure that terms corresponding to positions are also typable. This means that we cannot simply regard justification pointers as indicating parallel threads of computation and have to sequentialize threads where possible. The details of the adaptation are presented in Appendix ??. The example below illustrates this new definability algorithm.

Example 16. Let us consider a position in the game for $\mathbf{c o m}^{2} \rightarrow \mathbf{c o m}$ :

$$
\text { run } \cdot \operatorname{run}_{1} \cdot \operatorname{run}_{1} \cdot o k_{1} \cdot \operatorname{run}_{1} \cdot o k_{1} \cdot \operatorname{run}_{1} \cdot o k_{1} \cdot o k_{1} \cdot o k
$$


The algorithm from $[15,14]$ would return

$\lambda x$.newvar $x_{0}, x_{3}, x_{5}, x_{7}, x_{8}:=0$ in $x_{0}:=1 ; M ;$ WAIT $_{9}$,

where $M \equiv\left(P_{1}\left\|P_{2}\right\| P_{4} \| P_{6}\right)$ and

$$
\begin{aligned}
& P_{1} \equiv \text {WAIT }_{1} ; x ; x_{3}:=1, \quad P_{2} \equiv \text { WAIT }_{2} ; x ; x_{5}:=1, \\
& P_{4} \equiv \text { WAIT }_{4} ; x ; x_{8}:=1, \quad P_{6} \equiv \text { WAIT }_{6} ; x ; x_{7}:=1,
\end{aligned}
$$

but the term does not have the required type $\mathbf{c o m}^{2} \rightarrow \mathbf{c o m}$. The refined version produces $M \equiv\left(P_{1} ; P_{4}\right) \|\left(P_{2} ; P_{6}\right)$ instead. The term $W_{A I T}$ tests whether all variables $x_{j}$ with indices less than $i$ are set to 1 and diverges if they are not.

Consequently, the following properties can be proved as in [15].

Lemma 17. Suppose $\theta$ is a type with constraints $\eta$ and $s \in R_{\llbracket \theta \rrbracket_{\eta}}$. Then there exists a term $\vdash_{r} M: \theta$ such that $\llbracket M \rrbracket$ is the least saturated strategy containing $s$.

Lemma 18. Suppose $\Gamma \vdash_{r} M_{1}, M_{2}: \theta$ and let $\eta^{\mathrm{a}}$ be the final assignment of assumed bounds. $\Gamma \vdash M_{1} \check{r}_{r} M_{2}: \theta$ implies $\operatorname{comp}\left(\llbracket \Gamma \vdash_{r} M_{1}: \theta \rrbracket_{\eta^{\mathrm{a}}}\right) \subseteq \operatorname{comp}\left(\llbracket \Gamma \vdash_{r} M_{2}: \theta \rrbracket_{\eta^{\mathrm{a}}}\right)$.

Lemmas 15 and 18 imply full abstraction.

Theorem 19. Suppose $\Gamma \vdash_{r} M_{1}, M_{2}: \theta$ and let $\eta^{\text {a }}$ be the final assignment of assumed bounds.

- $\Gamma \vdash M_{1} \check{r}_{r} M_{2}: \theta \Longleftrightarrow \operatorname{comp}\left(\llbracket \Gamma \vdash_{r} M_{1}: \theta \rrbracket_{\eta^{\mathrm{a}}}\right) \subseteq \operatorname{comp}\left(\llbracket \Gamma \vdash_{r} M_{2}: \theta \rrbracket_{\eta^{\mathrm{a}}}\right)$.

- $\Gamma \vdash M_{1} \cong_{r} M_{2}: \theta \Longleftrightarrow \operatorname{comp}\left(\llbracket \Gamma \vdash_{r} M_{1}: \theta \rrbracket_{\eta^{\mathrm{a}}}\right)=\operatorname{comp}\left(\llbracket \Gamma \vdash_{r} M_{2}: \theta \rrbracket_{\eta^{\mathrm{a}}}\right)$

\section{Regular representation}

In this section we show sets of complete plays $\operatorname{comp}\left(\llbracket \Gamma \vdash_{r} M: \theta \rrbracket_{\eta^{\mathrm{a}}}\right)$ can be represented faithfully as regular languages and compared by checking language equivalence. The main difficulty to be addressed is the need to represent pointers.

For any bounded game $\theta$, we represent the positions of $R_{\llbracket \theta \rrbracket_{\eta^{\mathrm{a}} \eta^{\mathrm{g}}}}$ using the alphabet $\mathcal{A}(\theta)$ defined as follows:

$$
\begin{aligned}
\mathcal{A}(\beta) & =M_{\llbracket \beta \rrbracket}, \\
\mathcal{A}(\gamma \rightarrow \theta) & =\mathcal{A}(\gamma)+\mathcal{A}(\theta), \\
\mathcal{A}\left(\theta^{n}\right) & =\left\{m^{i} \mid m \in \mathcal{A}(\theta), 1 \leqslant i \leqslant n\right\} .
\end{aligned}
$$

Thus, elements of $\mathcal{A}(\theta)$ can be seen as moves of $\llbracket \theta \rrbracket$ decorated with a vector $\vec{i}=\left(i_{1}, \ldots, i_{k}\right)$ of labels produced by the last clause. The letters $m^{\vec{i}}$ will be used to encode occurrences of $m$ in positions from $R_{\llbracket \theta \rrbracket_{\eta^{\mathrm{a}} \eta_{\mathrm{g}}^{\mathrm{g}}}}$ subject to two invariants.

- If a question $q$ has several open occurrences then each of them will be represented by a different vector.

- Suppose an occurrence of a question $q$ is represented by $q^{\vec{i}}$. If an occurrence of another question $m$ is justified by the above occurrence of $q$, then $m$ is represented as $m^{j i}$ for some $j \in \mathbb{N}$.

We explain below how each position from the game under question will be represented so that the invariants are satisfied and only letters from $\mathcal{A}(\theta)$ are used. Note that the initial moves of $\theta$ occur without labels in $\mathcal{A}(\theta)$. They will also be represented as such in positions (this never leads to ambiguities since positions have unique initial moves). Given a representation of $s$ a representation of $s m$ is calculated as follows.

- If $m$ is an answer to an occurrence of $q$ in $s$ represented by $q^{\vec{i}}$ then $m$ is represented by $m^{\vec{i}}$.

- If $m$ is a question justified by an occurrence of $q$ in $s$ represented by $q^{\vec{i}}$, then there exists a sub-game $G_{m}^{n} \multimap G_{q}$ of $\llbracket \theta \rrbracket_{\eta^{\mathrm{a}} \eta^{\mathrm{g}}}$ such that $q, m$ are initial moves of, respectively, $G_{q}, G_{m}$. Since $s m$ is a position of $\llbracket \theta \rrbracket_{\eta^{\mathrm{a}} \eta^{\mathrm{g}}}$ there can be at 
most $n-1$ open questions in $s$ that are justified by the same occurrence of $q$ and, hence, represented by $q^{j \vec{i}}$. Thus one of the labels from $\{1, \ldots, n\}$, say $k$, has not been used. Then we represent $m$ as $m^{k i}$ (any such $k$ will do).

Note that, thanks to the labels, justification pointers can be uniquely reconstructed from the representation, so it is faithful. However, it is not unique because of the arbitrary choice of $k$. We will say that a representation is canonical if $k$ is always chosen to be the least $k$ available. The notion of canonicity is crucial to comparing representations of positions as they will provide the link between language equivalence and program equivalence.

Given a set $S$ of strings over $\mathcal{A}(\theta)$ representing a set of plays (e.g. a strategy) on $R_{\llbracket \theta \rrbracket_{\eta^{a} \eta^{\mathrm{g}}}}$ we write $\operatorname{can}(S)$ for the canonization of that representation.

Lemma 20. If $S$ is regular so is can $(S)$.

Proof. Given an automaton accepting $S$ one construct one for $\operatorname{can}(S)$. The number of open questions in any position of $R_{\llbracket \theta \rrbracket_{\eta^{\mathrm{a}} \eta^{\mathrm{g}}}}$ is uniformly bounded. Hence, with the help of finite memory we can keep track of all labels of open questions during the runtime of the automaton and relabel the accepted letters as required in a canonical representation. Since only finite store is needed, all this can be done by a finite automaton, so $\operatorname{can}(S)$ is also regular. The formal construction proceeds by annotating the states of the original automaton with all possible configurations of the finite memory. A possible version is shown below.

Let $\left\langle Q, z_{0}, \delta, F\right\rangle$ be the automaton accepting $S$. Then we define $\left\langle Q^{\prime}, z_{0}^{\prime}, \delta^{\prime}, F^{\prime}\right\rangle$ as follows. Let $\mathcal{M}=\{(m, \vec{i}, \vec{j}) \mid$ $m^{\vec{i}}, m^{\vec{j}} \in \mathcal{A}(\theta), m$ is a question $\}$ and $Q^{\prime}=Q \times \wp_{B}(\mathcal{M})$ where $\wp_{B}(\mathcal{M})$ stands for the set of subsets of $\mathcal{M}$ of size at most $B$ and $B$ is the uniform bound on the number of open questions in $R_{\llbracket \theta \rrbracket_{\eta^{\mathrm{a}} \eta^{\mathrm{g}}}}$. Let $z_{0}^{\prime}=\left(z_{0}, \emptyset\right)$ and $F^{\prime}=F \times\{\emptyset\}$.

- If $\delta(z, q)=z^{\prime}$ then define $\delta^{\prime}((z, \emptyset), q)=\left(z^{\prime},\{(q,(),())\}\right)$.

- If $\delta\left(z, a^{\vec{i}}\right)=z^{\prime}$ then for all $q, \vec{h}, X \in \wp_{B}(\mathcal{M})$ such that $(q, \vec{i}, \vec{h}) \in X$ and $q \vdash a$ include $\left(z^{\prime}, X \backslash\{(q, \vec{i}, \vec{h})\}\right)$ in $\delta^{\prime}\left((z, X), a^{\vec{h}}\right)$.

- If $\delta\left(z, q^{j \vec{i}}\right)=z^{\prime}$ then for all $q_{1}, \vec{h}, X \in \wp_{B}(\mathcal{M})$ such that $\left(q_{1}, \vec{i}, \vec{h}\right) \in X$ and $q_{1} \vdash q$ include $\left(z^{\prime}, X^{\prime}\right)$ in $\delta^{\prime}\left((z, X), q^{k \vec{h}}\right)$ provided $\{1, \ldots, k-1\} \subseteq U, k \notin U$, where $U=\left\{u \mid \exists_{\vec{g}, q_{2}}\left(\left(q_{2}, \vec{g}, u \vec{h}\right) \in X\right.\right.$ and $\left.\left.q_{1} \vdash q_{2}\right)\right\}, X^{\prime}=X \cup\{(q, j \vec{i}, k \vec{h})\}$ and $\left|X^{\prime}\right| \leqslant B$.

Theorem 21. The canonical representation of $\operatorname{comp}\left(\llbracket \Gamma \vdash_{r} M: \theta \rrbracket_{\eta^{\mathrm{a}}}\right)$, denoted simply by $\llbracket \Gamma \vdash_{r} M: \theta \rrbracket$ below, is a regular language over

$$
\mathcal{A}=\mathcal{A}\left(\theta_{1}^{n_{1}}\right)+\cdots+\mathcal{A}\left(\theta_{k}^{n_{k}}\right)+\mathcal{A}(\theta) .
$$

Proof. Many of the definitions for the imperative part of the language have the same flavour as those for Idealized Algol [13]. Sometimes the operation on regular languages will have to be followed by an explicit conversion to canonical form.

We define the $(\Gamma \vdash M \mid$ notations by the following decompositions:

$$
\begin{aligned}
& \llbracket \Gamma \vdash_{r} M: \mathbf{c o m} \rrbracket=\operatorname{run} \cdot\left(\Gamma \vdash_{r} M\right) \cdot o k \\
& \llbracket \Gamma \vdash_{r} M: \exp \rrbracket=\sum_{i=0}^{\operatorname{MAX}} q \cdot\left(\Gamma \vdash_{r} M D_{i} \cdot i\right. \\
& \llbracket \Gamma \vdash_{r} M: \operatorname{var} \rrbracket=\sum_{i=0}^{\operatorname{MAX}} \text { write }(i) \cdot\left(\Gamma \vdash_{r} M\right\rangle_{i}^{w} \cdot o k+\sum_{i=0}^{\mathrm{MAX}} \mathrm{read} \cdot\left(\left|\Gamma \vdash_{r} M\right\rangle_{i}^{r} \cdot i\right. \\
& \llbracket \Gamma \vdash_{r} M: \mathbf{s e m} \rrbracket=g r a b \cdot\left(\Gamma \vdash_{r} M\right\rangle^{g} \cdot o k+\text { release } \cdot\left(\Gamma \vdash_{r} M\right\rangle^{r} \cdot o k
\end{aligned}
$$

It is convenient to define $\llbracket \Gamma \vdash_{r} M \rrbracket$ via $\left(\Gamma \vdash_{r} M \mid\right)$ :

$$
\left(\Gamma \vdash_{r} M ; N D=\left(\Gamma \vdash_{r} M|\cdot| \Gamma \vdash_{r} N D\right.\right.
$$




$$
\begin{aligned}
& \left(\Gamma \vdash_{r} \text { if } M \text { then } N_{1} \text { else } N_{2} D=\left(\Gamma \vdash_{r} M D_{0} \cdot \mid \Gamma \vdash_{r} N_{2} D+\left(\sum_{i=1}^{\mathrm{MAX}}\left(\Gamma \vdash_{r} M D_{i}\right) \cdot \mid \Gamma \vdash_{r} N_{1} D\right.\right.\right. \\
& \left(\Gamma \vdash_{r} \text { while } M \text { do } N D=\left(( \sum _ { i = 1 } ^ { \operatorname { M A X } } | \Gamma \vdash _ { r } M D _ { i } ) \cdot ( | N | ) ^ { * } \cdot \left(\mid \Gamma \vdash_{r} M D_{0}\right.\right.\right. \\
& \left(\Gamma \vdash_{r} ! M D_{i}=\left(\Gamma \vdash_{r} M D_{i}^{r}\right.\right. \\
& \left(\Gamma \vdash_{r} M:=N D=\sum_{i=0}^{\operatorname{MAX}}\left(\left(\Gamma \vdash_{r} N D_{i} \cdot\left(\mid \Gamma \vdash_{r} M\right)_{i}^{w}\right)\right.\right. \\
& \left(\Gamma \vdash_{r} \operatorname{grab}(M)\right)=\left(\Gamma \vdash_{r} M\right)^{g} \\
& \left(\Gamma \vdash_{r} \text { release }(M)\right)=\left(\Gamma \vdash_{r} M\right)^{r}
\end{aligned}
$$

The above cases do not require explicit canonization. Neither does that of $\lambda$-abstraction which is interpreted using the appropriate associativity isomorphism of the disjoint sum.

For semaphore or variable binding it suffices to consider the histories in which the moves occur completely sequentially (in a canonical representation they are labelled with 1) [15]. We define

$$
\text { cell }_{m}=\left(\operatorname{read}^{1} \cdot m^{1}\right)^{*} \cdot\left(\sum_{i=0}^{\operatorname{MAX}}\left(\text { write }(i)^{1} \cdot o k^{1} \cdot\left(\operatorname{read}^{1} \cdot i^{1}\right)^{*}\right)\right)^{*}
$$

and

$$
\llbracket \Gamma \vdash_{r} \text { newvar } x:=m \text { in } M: \beta \rrbracket=\left(\llbracket \Gamma, x: \operatorname{var}^{n} \vdash M \rrbracket \cap \overline{\operatorname{cell}_{m}}\right) \backslash \mathcal{A}\left(\operatorname{var}^{n}\right),
$$

where $\bar{E}=E \amalg(\mathcal{A}(\Gamma)+\mathcal{A}(\beta))^{*}$ and $L \backslash A$ is obtained by erasing the symbols from $A$ in strings from $L$. Similarly, let us define $G=g r a b^{1} \cdot o k^{1}$ and $R=$ release $^{1} \cdot o k^{1}$. Then

$$
\begin{aligned}
& \llbracket \Gamma \vdash_{r} \text { newsem } x:=0 \text { in } M: \beta \rrbracket=\left(\llbracket \Gamma, x: \mathbf{s e m}^{m} \vdash M \rrbracket \cap \overline{(G \cdot R)^{*} \cdot(G+\varepsilon)}\right) \backslash \mathcal{A}\left(\mathbf{s e m}^{m}\right) \\
& \llbracket \Gamma \vdash_{r} \text { newsem } x:=1 \text { in } M: \beta \rrbracket=\left(\llbracket \Gamma, x: \mathbf{s e m}^{m} \vdash_{r} M \rrbracket \cap \overline{(R \cdot G)^{*} \cdot(R+\varepsilon)}\right) \backslash \mathcal{A}\left(\mathbf{s e m}^{m}\right) .
\end{aligned}
$$

We can take $\left.\left(\Gamma, \Delta \vdash_{r} M \| N\right\rangle\right)$ to be $\left(\Gamma \vdash_{r} M D U\left(\Delta \vdash_{r} N D\right)\right.$, which preserves canonicity.

Contraction is defined through renaming of labels associated with $y$. The labels $1, \ldots, n$ are replaced with $m+$ $1, \ldots, m+n$. This induces a homomorphism on the language so the result is still regular but needs canonization.

We write $\mathrm{id}_{\theta}$ for $\llbracket x: \theta \vdash_{r} x: \theta \rrbracket$. id $\mathrm{id}_{\text {com }}$ is defined by $\left\{r u n \cdot r u n^{1} \cdot o k^{1} \cdot o k\right\}$. For other base types the definition is analogous [13]. We extend it to function types $\theta^{n} \rightarrow \theta^{\prime}$ as follows. Let $\operatorname{id}_{\theta^{\prime}}=\sum_{q, a}\left(q \cdot q^{1} \cdot \mathrm{id}_{\theta^{\prime}}^{q, a} \cdot a^{1} \cdot a\right)$. Then

$$
\operatorname{id}_{\theta^{n} \rightarrow \theta^{\prime}}=\operatorname{can}\left(\sum_{q, a}\left(q \cdot q^{1} \cdot\left(\amalg_{i=1}^{n}\left(\operatorname{id}_{\theta}^{i 1}\right)^{*} \amalg \operatorname{id}_{\theta^{\prime}}^{q, a}\right) \cdot a^{1} \cdot a\right)\right),
$$

where $\operatorname{id}_{\theta}^{j 1}$ is $\operatorname{id}_{\theta}$ in which each move $m^{\vec{i}}$ is replaced with $m^{\vec{i} j 1}$ if it comes from the right copy of $\theta$ and with $m^{\vec{i} j}$ if it comes from the left one.

For application it is crucial that canonical representations interact as the interaction has to be represented in the same way both by the function and by the argument. Let $\Delta=\theta_{1}^{n_{1}}, \ldots, \theta_{k}^{n_{k}}$. For $i=1, \ldots, n$ let $\tilde{N}_{i}$ be the same as $\llbracket \Delta \vdash_{r} N: \theta \rrbracket$ except that the moves from the $\theta$-component are additionally decorated with the label $i$ while the original labels of moves from $\theta_{j}(1 \leqslant j \leqslant k)$ (i.e. $\left.1, \ldots, n_{j}\right)$ are replaced, respectively, with $(i-1) n_{j}+1, \ldots, i n_{j}$. Clearly, these operations preserve regularity. Then we can define $\llbracket \Gamma, \Delta \vdash M N: \theta^{\prime} \rrbracket$ to be $\operatorname{can}\left((\tilde{M} \cap \tilde{N}) \backslash \mathcal{A}\left(\theta^{n}\right)\right)$ where

$$
\begin{aligned}
\tilde{M} & =\llbracket \Gamma \vdash M: \theta^{n} \rightarrow \theta^{\prime} \rrbracket \amalg \mathcal{A}\left(\theta_{1}^{n \cdot n_{1}}\right)^{*} \amalg \cdots \amalg \mathcal{A}\left(\theta_{k}^{n \cdot n_{k}}\right)^{*} \\
\tilde{N} & =\mathcal{A}(\Gamma)^{*} \amalg \operatorname{can}\left(\amalg_{i=1}^{n}\left(\tilde{N}_{i}\right)^{*}\right) \amalg \mathcal{A}\left(\theta^{\prime}\right)^{*} .
\end{aligned}
$$

Finally, no changes are needed to interpret subsumption.

Theorem 22. $\check{\sim}_{r}$ and $\cong_{r}$ are decidable. 


\section{Further work}

We have already stated that we plan to study the syntactic properties of the system separately. The previous section establishes that there is a finite-state representation of terms of SCC, and that it can be used, in principle, for model checking using a method similar to [2]. Lemma 10 and the various examples we give suggest that the restrictions imposed by the tighter typing discipline are not onerous. However, to claim a fully automated verification (and certification) procedure the issue of automated type inference must be investigated. Finally, only by incorporating these theoretical results in a model-checking tool can we evaluate the practicality of the method.

\section{Acknowledgements}

This work was funded by British EPSRC (GR/R88861/01), Canadian NSERC and St. John's College, Oxford.

\section{Appendix A. Resource-sensitive definability}

We define a recursive algorithm, called $P R O C^{+}$, which takes a position $s$ in $R_{\llbracket \theta \rrbracket_{\eta^{a} \eta^{\mathrm{g}}}}$ and returns a term $\vdash_{r} P_{S}: \theta$ such that $\llbracket P_{S} \rrbracket$ is the least saturated strategy containing $s . P R O C^{+}$relies on a recursive procedure $P R O C$ which takes the original position as the initial argument. In the recursive invocations of $P R O C$, the argument is a subsequence of the form $s\lceil m$, where $t\lceil m$ is the subsequence of $t$ consisting of $m$ and all moves hereditarily justified by $m$, always an O-question. Note that consequently a move in $t$ is answered in $t$ iff it is answered in $s$.

Throughout the execution of $P R O C$ it is convenient to use indices relative to the original $s$; we write $s_{i}$ for the $i$ th move of $s$, assuming $s_{0}$ initial. In order to generate the desired position we need to control the way in which both $\mathrm{P}$ and $\mathrm{O}$ move. We control P-moves using guards that wait for special side-effects (time-stamps) caused by O-moves. The effects take place only if a correct O-move is played and we make sure that they occur only once by using a fresh semaphore for each O-move. This allows us to enforce arbitrary synchronization policies, restricting the order of moves present in the original sequence up to the reorderings dictated by the saturation conditions. To that effect, a global variable $x_{j}$, i.e. a variable which is bound by new at the top level and initialized to 0 , is associated which each index of an $\mathrm{O}$ move in $s$. The time-stamp consists of assigning 1 to the variable, $x_{j}:=1$.

For $1 \leqslant j \leqslant|s|-1$, let us define:

$$
O_{j}=\left\{i \in \mathbb{N} \mid 0 \leqslant i<j, s_{i} \text { is an O-move }\right\} .
$$

We define $W I_{j}$ as the term which checks for time-stamps originating from all the O-moves with indices smaller than $j$ :

$$
W I T_{j} \equiv\left[\bigwedge_{g \in O_{j}}\left(! x_{g}=1\right)\right] .
$$

$\operatorname{PROC}(t: \theta)$ where $\theta=\theta_{1}^{n_{1}} \rightarrow \cdots \rightarrow \theta_{h}^{n_{h}} \rightarrow \beta$ is defined as follows in two stages which manage O-questions and $\mathrm{P}$-answers, and, respectively, P-questions and O-answers.

If $t$ is empty, $\lambda p_{1} \cdots p_{h} . \Omega_{\theta_{0}}$ is returned. Otherwise, let $o=s_{i}$ be the initial move of $t$ (which is always an O-question).

(1) For $a=1, \ldots, h$ let $i_{1}^{a}<\cdots<i_{m_{a}}^{a}$ be the $s$-indices of all occurrences of questions from $\theta_{a}$ explicitly justified by $s_{i}$. We define a function $\phi:\left\{i_{1}^{a}, \ldots, i_{m_{a}}^{a}\right\} \rightarrow\left\{1, \ldots, n_{a}\right\}$ (which will assign moves to threads) inductively using the ordering $i_{1}^{a}<\cdots<i_{m_{a}}^{a}$. Consider $i_{c}^{a}$. Then at most $n_{a}-1$ out of $s_{i_{1}^{a}}, \ldots, s_{i_{c-1}^{a}}$ are open in $s \leqslant i_{c}^{a}$. Let $Q_{c}^{\text {op }}$ be the set containing them (i.e. $\left|Q_{c}^{\mathrm{op}}\right|<n_{a}$ ). Define $\phi\left(i_{c}^{a}\right)$ to be the least number from $\left\{1, \ldots, n_{a}\right\}$ different from each $\phi(m)$ $\left(m \in Q_{c}^{\mathrm{op}}\right)$. Assume we have terms $P_{i_{d}^{a}}: \operatorname{com}\left(d=1, \ldots, m_{a}\right)$ to be defined later. For $k=1, \ldots, n_{a}$ let $R_{a}^{k}$ be the sequential composition of all $P_{i_{d}^{a}}$ such that $\phi\left(i_{d}^{a}\right)=k$ (ordered in the same way as $i_{d}^{a}$ 's). Then let $R_{a}=R_{a}^{1}\|\cdots\| R_{a}^{n_{a}}$. $P R O C$ returns the following results, depending on $\beta$.

- $\beta=$ com:

$$
\lambda p_{1} \cdots p_{h} \cdot\left(x_{i}:=1\right) ;\left(R_{1}\|\cdots\| R_{h}\right) ; P A N S_{i}^{\mathbf{c o m}},
$$




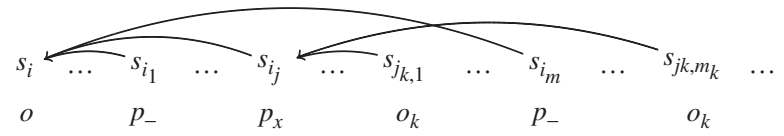

Fig. A.1. Questions and justification pointers.

where

$$
\operatorname{PANS}_{i}^{\mathbf{c o m}} \equiv \begin{cases}\Omega_{\text {com }} & s_{i} \text { is unanswered in } t \\ W_{A I T} i^{\prime} & s_{i^{\prime}} \text { answers } s_{i} \text { in } t .\end{cases}
$$

By convention, $\left(R_{1}\|\cdots\| R_{h}\right)$ degenerates to skip for $m=0$.

- $\beta=$ exp: Same as for com except that $P A N S_{i}^{\text {com }}$ is replaced with $P A N S_{i}^{\text {exp }}$ defined below.

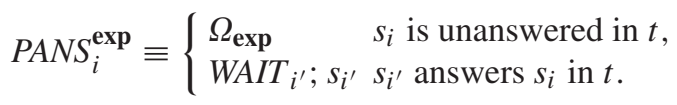

- $\beta=$ var:

○ If $s_{i}=$ read:

$$
\operatorname{mkvar}\left(\lambda x . \Omega_{\mathbf{c o m}},\left(x_{i}:=1\right) ;\left(R_{1}\|\cdots\| R_{h}\right) ; P A N S_{i}^{\exp }\right) .
$$

○ If $s_{i}=$ write $(v)$ :

$$
\operatorname{mkvar}\left(\lambda x \text {.if }(x=v) \text { then } x_{i}:=1 ;\left(R_{1}\|\cdots\| R_{h}\right) ; P A N S_{i}^{\mathbf{c o m}}, \Omega_{\exp }\right) .
$$

The presence of the $x=v$ test serves to ensure that the only acceptable move by $\mathrm{O}$ is only that which writes $v$, and no other value.

- $\beta=\mathbf{s e m}$ is analogous to var:

○ If $s_{i}=g r a b$ :

$$
\operatorname{mksem}\left(\left(x_{i}:=1\right) ;\left(R_{1}\|\cdots\| R_{h}\right) ; \operatorname{PANS}_{i}^{\mathrm{com}}, \Omega_{\mathrm{com}}\right) .
$$

○ If $s_{i}=$ release:

$$
\operatorname{mksem}\left(\Omega_{\text {com }},\left(x_{i}:=1\right) ;\left(R_{1}\|\cdots\| R_{h}\right) ; \text { PANS }_{i}^{\mathrm{com}}\right) .
$$

(2) Let $i_{1}<\cdots<i_{m}$ be the $s$-indices of all occurrences of questions justified by $s_{i}$. Here we show how to define the terms $P_{i_{j}}$ for $1 \leqslant j \leqslant m$. Let us fix $j$ and suppose that $s_{i_{j}}=p_{x}(1 \leqslant x \leqslant h)$ and $\theta_{x}=\theta_{1}^{\prime m_{1}} \rightarrow \cdots \rightarrow \theta_{n}^{\prime m_{n}} \rightarrow \beta^{\prime}$. Let $o_{1}, \ldots, o_{n}$ be all the O-questions enabled by $p_{x}$ (corresponding to $\theta_{1}^{\prime}, \ldots, \theta_{n}^{\prime}$, respectively).

For each $k(1 \leqslant k \leqslant n)$ let $j_{k, 1}<\cdots<j_{k, m_{k}}$ be the $s$-indices of all occurrences of $o_{k}$ in $t$ which are explicitly justified by $s_{i j}$ (see Fig. A.1).

If $m_{k}=0$, then $P_{j}^{k} \equiv \Omega_{\theta_{k}^{\prime}}$. Otherwise, for all $l=1, \ldots, m_{k}$ we make the following definitions: $P_{j}^{k, l} \equiv$ $\operatorname{PROC}\left(t\left\lceil s_{j_{k, l}}: \theta_{k}^{\prime}\right)\right.$ and

$$
P_{j}^{k} \equiv O N C E_{w_{j_{k, 1}}}\left[P_{j}^{k, 1}\right] \text { or } \cdots \text { or } O N C E_{w_{j_{k, m_{k}}}}\left[P_{j}^{k, m_{k}}\right],
$$

where $w_{j_{k, 1}}, \ldots, w_{j_{k, m_{k}}}$ are fresh semaphore names and $O N C E_{w}(M)=\operatorname{grab}(w) ; M$. Finally, we define the terms $P_{i_{j}}$, depending on $\beta^{\prime}$. The fresh variables $z_{c}$ are used to "store" O-answers for future tests.

First, it is useful to define the following macros:

$$
\begin{aligned}
O A N S_{c}^{\text {com }} & \equiv \begin{cases}\text { skip } & s_{c} \text { is unanswered in } t, \\
x_{c^{\prime}}:=1 & s_{c^{\prime}} \text { answers } s_{c} \text { in } t,\end{cases} \\
O A N S_{c}^{\text {exp }} & \equiv \begin{cases}\text { skip } & s_{c} \text { is unanswered in } t \\
\text { if }\left(! z_{c}=s_{c^{\prime}}\right) \text { then } x_{c^{\prime}}:=1 \text { else skip } & s_{c^{\prime}} \text { answers } s_{c} \text { in } t .\end{cases}
\end{aligned}
$$

- For $\beta^{\prime}=\mathbf{c o m}, P_{i_{j}} \equiv W A I T_{i_{j}} ;\left(p_{x} P_{j}^{1} \cdots P_{j}^{n}\right) ; O A N S_{i_{j}}^{\text {com }}$ 
- For $\beta^{\prime}=\exp , P_{i_{j}} \equiv W A I T_{i_{j}} ; z_{i_{j}}:=\left(p_{x} P_{j}^{1} \cdots P_{j}^{n}\right) ; O A N S_{i_{j}}^{\exp }$.

- For $\beta^{\prime}=$ var there are two sub-cases:

○ If $s_{i_{j}}=\mathrm{read}, P_{i_{j}} \equiv$ WAIT $_{i_{j}} ; z_{i_{j}}:=!\left(p_{x} P_{j}^{1} \cdots P_{j}^{n}\right) ; O A N S_{i_{j}}^{\exp }$.

○ If $s_{i_{j}}=$ write $(v), P_{i_{j}} \equiv$ WAIT $_{i_{j}} ;\left(p_{x} P_{j}^{1} \cdots P_{j}^{n}\right):=v ; O A N S_{i_{j}}^{\text {com }}$.

- For $\beta^{\prime}=\mathbf{s e m}, P_{i_{j}}$ there are two sub-cases:

○ If $s_{i_{j}}$ is $\operatorname{grab}, P_{i_{j}} \equiv$WAIT $_{i_{j}} ; \operatorname{grab}\left(p_{x} P_{j}^{1} \ldots P_{j}^{n}\right) ; O A N S_{i_{j}}^{\text {com }}$

○ If $s_{i_{j}}$ is release, $P_{i_{j}} \equiv W A I T_{i_{j}}$; release $\left(p_{x} P_{j}^{1} \cdots P_{j}^{n}\right) ; O A N S_{i_{j}}^{\text {com }}$.

After $\operatorname{PROC}(s: \theta)$ returns $\lambda p_{1} \cdots p_{k} . M$, all variables and semaphores used in the construction of $M$ (i.e. $x_{-}, z_{-}, w_{-}$) must be bound at the topmost level (the variables $x_{-}$must be initialized to 0 , the semaphores $w_{-}$to 0 , the initial values of $z_{-}$are irrelevant). For $\beta=\mathbf{c o m}$, exp this is done by taking

$$
\lambda p_{1} \cdots p_{k} \text { newvar } \vec{x}, \vec{z}:=\overrightarrow{0} \text { in (newsem } \vec{w}:=\overrightarrow{0} \text { in } M \text { ). }
$$

For $\beta=$ var, com the binders have to be pushed inside mkvar or mksem. We denote the final term by $\operatorname{PROC}^{+}(s: \theta)$.

\section{References}

[1] S. Abramsky, Beyond full abstraction: model-checking for Algol-like languages, series of lectures given at the Marktoberdorf Summer School, 2001.

[2] S. Abramsky, D.R. Ghica, A.S. Murawski, C.-H.L. Ong, Applying game semantics to compositional software modelling and verification, in: Proc. TACAS, Lecture Notes in Computer Science, Vol. 2988, Springer, Berlin, 2004, pp. 421-435.

[3] S. Abramsky, K. Honda, G. McCusker, Fully abstract game semantics for general references, in: Proc. IEEE Symp. Logic in Computer Science, Computer Society Press, Rockville, MD, 1998, pp. 334-344.

[4] S. Abramsky, R. Jagadeesan, P. Malacaria, Full abstraction for PCF, Inform. and Comput. 163 (2000) 409-470.

[5] S. Abramsky, G. McCusker, Linearity, sharing and state: a fully abstract game semantics for Idealized Algol with active expressions, in: P.W. O’Hearn, R.D. Tennent (Eds.), Algol-like Languages, Birkhaüser, Basel, 1997, pp. 297-329.

[6] S. Abramsky, G. McCusker, Full abstraction for Idealized Algol with passive expressions, Theoret. Comput. Sci. 227 (1999) 3-42.

[7] R. Alur, T.A. Henzinger, O. Kupferman, Alternating-time temporal logic, J. ACM 49 (5) (2002) 672-713.

[8] H.P. Barendregt, Lambda calculi with types, in: S. Abramsky, D.M. Gabbay, T.S.E. Maibaum (Eds.), Background: Computational Structures Handbook of Logic in Computer Science, Vol. 2, Oxford University Press, Oxford, England, 1992, pp. 117-309.

[9] S. Brookes, The essence of Parallel Algol, in: Proc. 11th Symp. Logic in Computer Science, 1996, pp. 164-173 (also published as Chapter 21 of [30]).

[10] D.R. Ghica, Regular-language semantics for a call-by-value programming language, in: Proc. MFPS, Electronic Notes in Computer Science, Vol. 45, Elsevier, Amsterdam, 2001.

[11] D.R. Ghica, A regular-language model for Hoare-style correctness statements, in: Proc. Verification and Computational Logic Workshop, Florence, Italy, 2001.

[12] D.R. Ghica, A games-based foundation for compositional software model checking, Ph.D. thesis, Queen's University School of Computing, Kingston, Ontario, Canada, also available as Oxford University Computing Laboratory Research Report RR-02-13, November 2002.

[13] D.R. Ghica, G. McCusker, Reasoning about Idealized Algol using regular expressions, in: Proc. ICALP, Lecture Notes in Computer Science, Vol. 1853, Springer, Berlin, 2000, pp. 103-115.

[14] D.R. Ghica, A.S. Murawski, Angelic semantics of fine-grained concurrency, Technical Report PRG-RR-03-20, Oxford University Computing Laboratory, available from http://users.ox.ac.uk/ coml $0074 /$ papers/asfgc.pdf, 2003.

[15] D.R. Ghica, A.S. Murawski, Angelic semantics of fine-grained concurrency, in: Proc. FOSSACS, Lecture Notes in Computer Science, Vol. 2987, Springer, Berlin, 2004, pp. 211-225 (an extended version is available as [14]).

[16] M. Hofmann, Linear types and non-size-increasing polynomial time computation, in: Proc. 14th Symp. Logic in Computer Science, IEEE, New York, 1999, pp. 464-473.

[17] M. Hofmann, A type system for bounded space and functional in-place update, Nordic J. Comput. 7 (4) (2002) 258-289.

[18] J. Hughes, L. Pareto, Recursion and dynamic data-structures in bounded space: towards embedded ML programming, ACM SIGPLAN Notices 34 (9) (1999) 70-81 (Proc. ICFP).

[19] J.M.E. Hyland, C.-H.L. Ong, On full abstraction for PCF: I. models, observables and the full abstraction problem, II. dialogue games and innocent strategies, III. a fully abstract and universal game model, Inform. and Comput. 163 (2) (2000) 285-408.

[20] H. Jifeng, M.B. Josephs, C.A.R. Hoare, A theory of synchrony and asynchrony, in: Programming Concepts and Methods, Elsevier, Amsterdam, 1990, pp. 459-473.

[21] J. Laird, Full abstraction for functional languages with control, in: Proc. 12th IEEE Symp. Logic in Computer Science, 1997 , pp. 58-67.

[22] J. Laird, A games semantics for idealized CSP, in Proc. 17th Annual Conf. Mathematical Foundations of Programming Semantics, Aarhus, Denmark, May 2001, Electronic Notes in Theoretical Computer Science, Elsevier, Amsterdam, pp. 157-176.

[23] P. Malacaria, C. Hankin, Generalized flowcharts and games (extended abstract), in: Proc. ICALP 1998, Lecture Notes in Computer Science, Vol. 1443, Springer, Berlin, 1998, pp. 363-374. 
[24] P. Malacaria, C. Hankin, Non-deterministic games and program analysis: an application to security, in: Proc. 14th IEEE Symp. Logic in Computer Science, IEEE, New York, 1999, pp. 443-452.

[25] G. McCusker, Games for recursive types, BCS Distinguished Dissertation, Cambridge University Press, Cambridge, 1998.

[26] M.L. Minsky, Computation: Finite and Infinite Machines, Prentice-Hall, Englewood Cliffs, NJ, 1967.

[27] A.S. Murawski, Functions with local state: regularity and undecidability, Theoret. Comput. Sci. 338 (1-3) (2005) 315-349.

[28] A. Mycroft, R. Sharp, A statically allocated parallel functional language, in: Proc. ICALP, Lecture Notes in Computer Science, Vol. 1853, 2000, pp. $37-48$.

[29] G.C. Necula, Proof-carrying code, in: Proc. 24th ACM Symp. Principles of Programming Languages, 1997, pp. $106-119$.

[31] C.-H.L. Ong, Observational equivalence of 3rd-order Idealized Algol is decidable, in: Proc. IEEE Symp. Logic in Computer Science, Computer Society Press, Rockville, MD, 2002, pp. 245-256.

[32] J.C. Reynolds, Syntactic control of interference, in: Proc. POPL, 1978, pp. 39-46.

[33] M. Tofte, Region inference for higher-order functional languages, in: Proc. SAS, Lecture Notes in Computer Science, Vol. 983, 1995, pp. 19-20.

[34] J.T. Udding, A formal model for defining and classifying delay-insensitive circuits and systems, Distributed Comput. 1 (4) (1986) $197-204$.

[35] K. Wansbrough, S.L.P. Jones, Once upon a polymorphic type, in: Proc. 26th ACM Symp. Principles of Programming Languages, 1999, pp. 15-28. 